\title{
REVIEW
}

\section{Emerging Concepts in Myeloid Cell Biology after Spinal Cord Injury}

\author{
Alicia L. Hawthorne and Phillip G. Popovich \\ Department of Neuroscience and Center for Brain and Spinal Cord Repair, The Ohio State University College of Medicine, 460 W.
} 12th Ave., 770 Biomedical Research Tower, Columbus, Ohio 43210

\begin{abstract}
Summary: Traumatic spinal cord injury (SCI) affects the activation, migration, and function of microglia, neutrophils and monocyte/macrophages. Because these myeloid cells can positively and negatively affect survival of neurons and glia, they are among the most commonly studied immune cells. However, the mechanisms that regulate myeloid cell activation and recruitment after SCI have not been adequately defined. In general, the dynamics and composition of myeloid cell recruitment to the injured spinal cord are consistent between mammalian species; only the onset, duration, and magnitude of the response vary. Emerging data, mostly from rat and mouse SCI models, indicate that resident and recruited myeloid cells are derived from multiple sources, including the yolk sac during development and the bone marrow and spleen in adulthood. After SCI, a complex array of chemokines and
\end{abstract}

cytokines regulate myelopoiesis and intraspinal trafficking of myeloid cells. As these cells accumulate in the injured spinal cord, the collective actions of diverse cues in the lesion environment help to create an inflammatory response marked by tremendous phenotypic and functional heterogeneity. Indeed, it is difficult to attribute specific reparative or injurious functions to one or more myeloid cells because of convergence of cell function and difficulties in using specific molecular markers to distinguish between subsets of myeloid cell populations. Here we review each of these concepts and include a discussion of future challenges that will need to be overcome to develop newer and improved immune modulatory therapies for the injured brain or spinal cord. Key Words: Monocytes, macrophages, neutrophils, microglia, cytokine, chemokine.

\section{INTRODUCTION}

Spinal cord injury (SCI) elicits a robust and persistent inflammatory response. This response, involving the activation of resident microglia and the infiltration of neutrophils, monocytes, and lymphocytes, has been characterized in a number of different species including humans. The breadth and potential impact of the different components of post-SCI inflammation, including post-injury activation of peripheral immune responses, have been recently reviewed [1-5]. Therefore, this review will focus on the development, trafficking, and activation of myeloid cells after SCI and will conclude with a brief overview of possible

Electronic supplementary material The online version of this article (doi:10.1007/s13311-011-0032-6) contains supplementary material, which is available to authorized users.

Address correspondence and reprint requests to: Phillip G. Popovich, $\mathrm{PhD}$, Department of Neuroscience and Center for Brain and Spinal Cord Repair, The Ohio State University College of Medicine, 460 W. 12th Ave., 770 Biomedical Research Tower, Columbus, OH 43210. E-mail: phillip.popovich@osumc.edu. therapeutic approaches for manipulating this distinct component of post-traumatic inflammation.

\section{Myeloid cells in inflammation}

Myeloid cells are a family of cells derived from hematopoietic progenitor cells and include monocytes, neutrophils, erythrocytes, and megakaryocytes (FIG. 1). Essentially, all leukocytes that cannot be characterized as lymphocytes are classified as myeloid cells. This review will focus on microglia, macrophages, and neutrophils because they are the main myeloid effector cells after SCI.

Within minutes of SCI, cells of the myeloid cell lineage become activated, creating a heterogeneous network of multifunctional cells capable of promoting injury and repair of neural tissue. Microglia are among the first myeloid cells to be set in motion, responding to changes in extracellular ions and ATP within minutes to hours post-injury [6-8]. Within the next few hours, neural and humoral signaling mechanisms elicit recruitment of cells from the circulation to the site of injury. Neutrophils arrive first, sometime between 3 to $24 \mathrm{~h}$ post-injury [9-11]. Monocytes arrive 


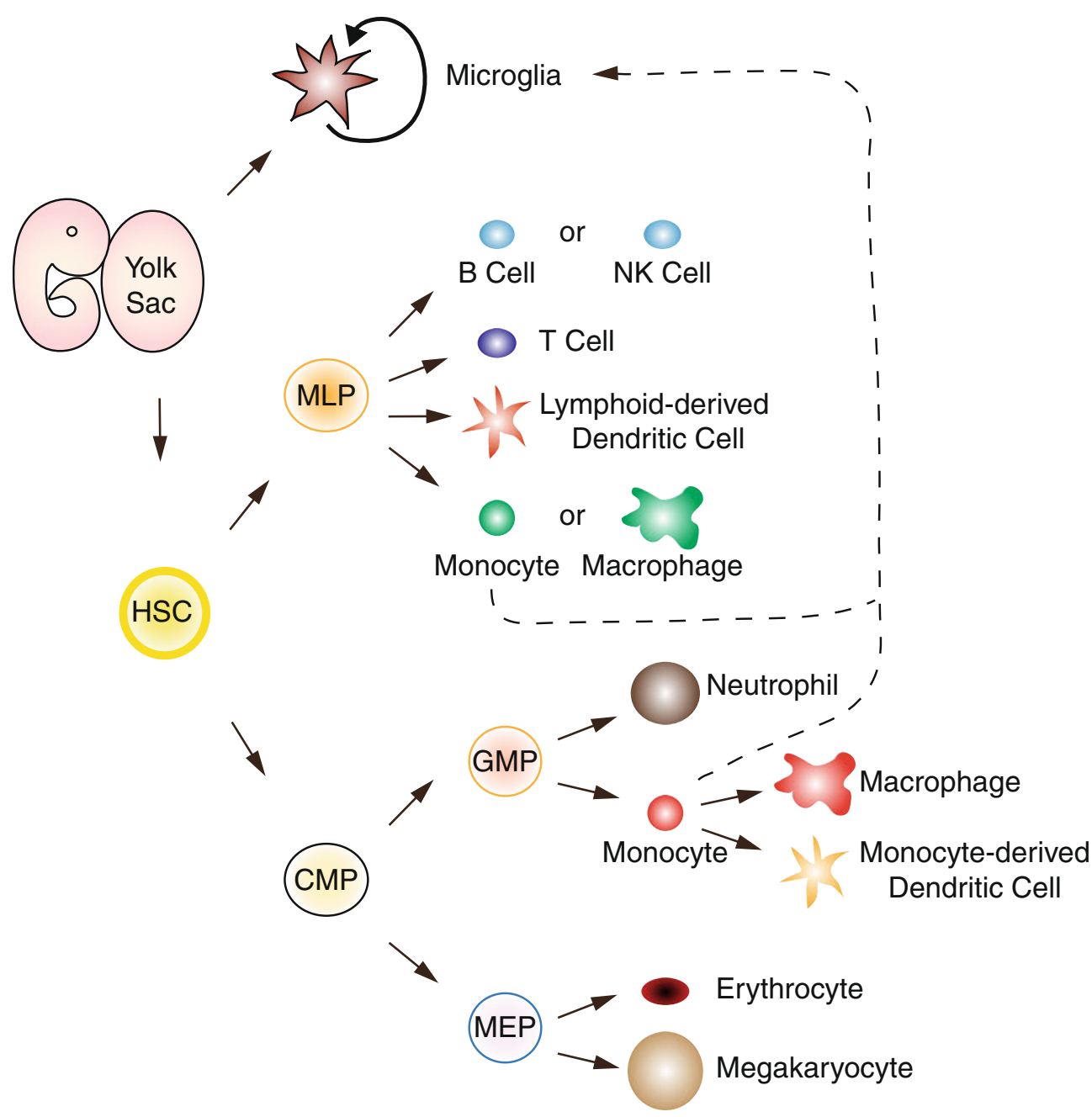

FIG. 1. Updated schematic of myelopoiesis. Microglia are resident central nervous system macrophages derived from myeloid progenitors originating in the yolk sac before embryonic day 8 . Adult microglia is an ontogenetically distinct population of macrophages that self renew. However, microglia may be repopulated from postnatal monocytes under certain circumstances (dashed line). Hematopoietic stem cells (HSCs) are also originally derived from the yolk sac and produce multi-lymphocyte progenitors (MLP) and common myeloid progenitors (CMP). MLPs give rise to lymphocytes ( $T$ and B) and NK cells, but also are capable of producing myeloid cells (e.g., monocytes or macrophages). CMPs, stimulated by a number of factors (see text), yield granulocyte-monocyte precursors (GMPs) and megakaryocyte-erythrocyte precursors (MEP). GMPs can be further stimulated to produce myeloid cells (e.g., neutrophils or monocytes). Monocytes differentiate into macrophages or monocyte-derived dendritic cells.

later, $\sim 2$ to 3 days post-injury (dpi) [11] with maximal infiltration occurring 1 to 2 weeks post-injury [9]. The dynamics and prevalence of intraspinal dendritic cells has not been well-defined, although cells expressing the dendritic cell antigen, integrin $\alpha \mathrm{E} 2$ antigen, infiltrate the injured rat spinal cord 3 to 7 days post-injury [12]. Similar to dendritic cells, myeloid-derived suppressor cells (MDSCs) have received little attention after spinal cord injury (SCI); however, in mice with experimental autoimmune encephalomyelitis (i.e., the animal model for multiple sclerosis) MDSCs infiltrate the inflamed central nervous system (CNS) [13].

Normally, inflammatory cascades are self-limiting and typically resolve within a matter of days to weeks.
However, within the injured spinal cord, neuroinflammatory cascades persist for extended periods of time. Activated macrophages and microglia are found in the injured spinal cord for at least 6 months in rats $[9,14]$, guinea pigs [15], and cats [16]. Neutrophils, although found in lesser numbers than monocytes, macrophages, and microglia, still persist for $\sim 6$ weeks post-injury in mice and $\sim 6$ months post-injury in rats $[9,17]$. In injured human spinal cords, defensin-positive neutrophil infiltrates persist for $\sim 10$ days after injury, whereas activated intraspinal macrophages and microglia can be found for at least 6 months post-injury [18]. Because the lifespan of neutrophils and macrophages is $\sim 5$ days or 2 months, respectively, it is likely that chemotactic gradients specific 


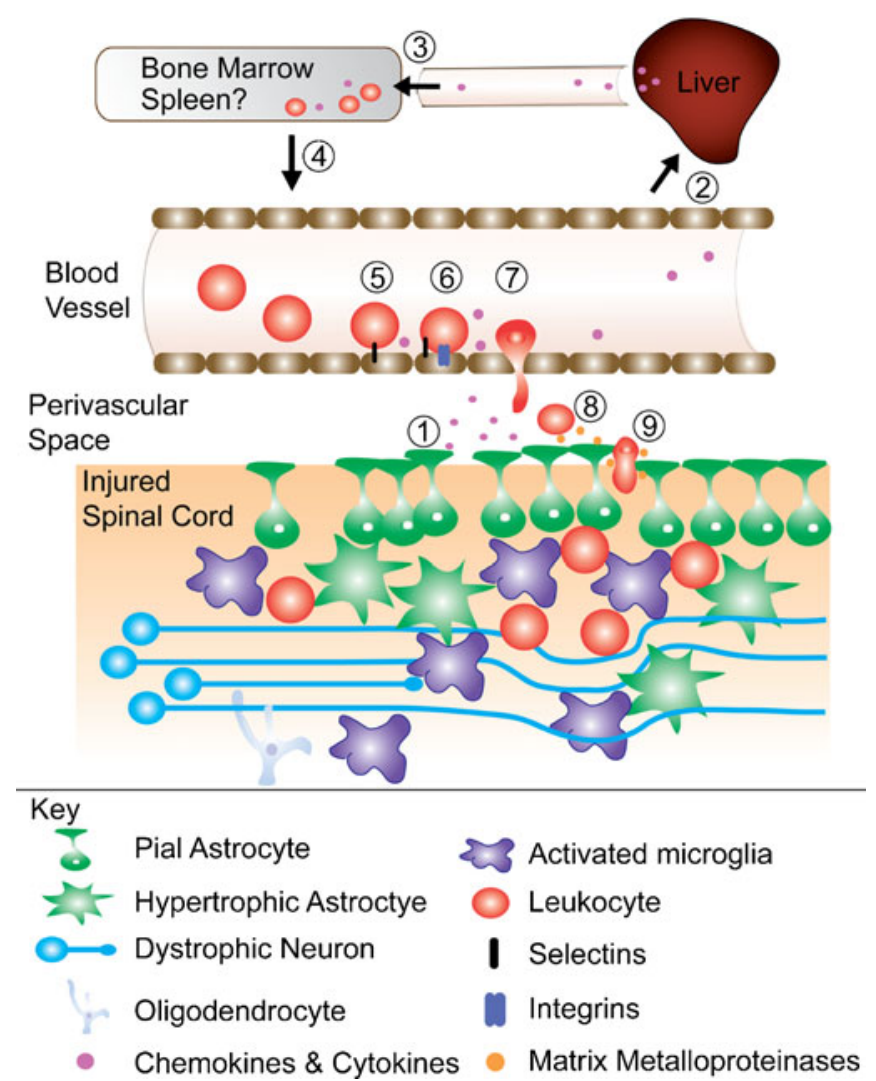

FIG. 2. Mechanisms of myeloid cell recruitment after spinal cord injury. (1) Injury to the spinal cord displaces pial glia, creating gaps in the glial limitans. Other astrocytes become reactive and hypertrophic. Neurons and oligodendrocytes are damaged, and microglia become reactive. Microglia, astrocytes, neurons, and oligodendrocytes synthesize and release chemokines and cytokines. (2) Chemokines and cytokines initiate chemokine synthesis in the liver. (3) Hepatic chemokines stimulate (4) myelopoiesis and the release of leukocytes from bone marrow (and maybe the spleen). (5) As they circulate, leukocytes bind to endothelia near the injury site first via selectins then (6) through stronger interactions with integrins. (7) Integrin binding allows firm attachment and margination with subsequent diapedesis through the blood-brain barrier into the perivascular space. (8) Leukocyte-derived matrix metalloproteinases (MMPs) help degrade the spinal basement membrane (9) allowing leukocytes to enter the spinal cord parenchyma.

for these myeloid cells persist indefinitely or recur periodically, allowing sustained recruitment of new cells to the injury site $[19,20]$.

The functional significance of resident and infiltrating myeloid cells in the injured spinal cord is controversial. Neutrophils can exacerbate hemorrhage, leading to more extensive pathology after SCI [21, 22]; however, these same cells may also trigger the onset of wound healing cascades in the injured spinal cord [23]. Similarly, microglia and macrophages can promote CNS repair (e.g., phagocytic removal of growth-inhibitory debris; stimulate neurite outgrowth) or exacerbate cell death and axonal degeneration at the site of injury [24-26]. The roles of dendritic cells and MDSCs are less welldefined. Dendritic cells may be beneficial after SCI, especially if they are prepared ex vivo and transplanted into the injury site, or if their function is augmented using exogenous growth factors/cytokines (e.g., granulocyte-macrophage colony stimulating factor [GMCSF]) $[27,28]$. MDSCs, if they are activated by SCI, could suppress deleterious autoimmune functions, including the activation of T-cells [29]. A functional role for MDSCs has not been characterized after SCI.

Damage caused by myeloid cells is not restricted to the spinal cord. Recent data show that myeloid cells activated as a result of SCI cause tissue damage in the liver and lungs [30]. Thus, activation of the immune system by SCI has long-lasting effects on the spinal cord and peripheral tissues.

\section{Myelopoiesis and sources of intraspinal myeloid cells after injury}

To fully appreciate the functional implications of myeloid cell recruitment and activation within the injured spinal cord, it is useful to know from where these cells originate and how and why they become activated. In embryos, hematopoiesis occurs in the yolk sac, then later, as the organism develops, in the liver, bone marrow, and spleen [31, 32] (FIG. 1). In classical models of hematopoiesis, a hematopoietic stem cell (HSC) gives 
rise to lineage-restricted precursors for lymphocytes or myeloid cells. This model has been called into question for mice because lymphocyte precursors were also found to give rise to myeloid cells [33-39]. Similarly, using human cells, recent data indicate that myeloid cell production is not limited to granulocyte-myeloid precursor cells $[39,40]$. Instead, HSCs give rise to common myeloid progenitors and multi-lymphoid progenitors (FIG. 1). Monocytes and macrophages can also be derived from either granulocyte-myeloid precursor cells or multi-lymphoid progenitors, although it is not clear yet if cells derived from these distinct progenitor cell populations differ in their tissue distribution or function.

Myelopoiesis is stimulated directly or indirectly by a wide range of cytokines and growth factors, including interleukin-1 (IL-1), IL-3, IL-6, IL-7, IL-11, granulocytecolony stimulating factor (G-CSF), granulocyte-monocytecolony stimulating factor (GM-CSF), monocyte-colony stimulating factor (M-CSF), stem cell factor (SCF), Flt3 ligand (Flt3-L), interferon-gamma (IFN- $\gamma$ ), and tumor necrosis factor (TNF- $\alpha$ ) [41-45]. When injected systemically, IL-7 increases the number of myeloid cells in the spleen and neutrophils and monocytes in the blood [41, 46, 47], presumably by increasing the mobilization of myeloid cells from sites of myelopoiesis [47, 48]. It is not known if circulating or cerebrospinal fluid levels of IL-7 increase after SCI; however, other pro-inflammatory cytokines (including IL-6 and TNF- $\alpha$ ) do increase in SCI animals and humans [49-55].

Microglia, the resident immune cells in the CNS, and the first myeloid cells to respond to SCI, are widely believed to be derived from circulating hematopoietic precursors, mostly blood monocytes that colonize the CNS during late stages of development [56-58]. However, recent data indicate that adult microglia arise instead from extra-embryonic yolk sac myeloid precursors during embryogenesis (about embryonic day 8) [31] (FIG. 1). Accordingly, adult microglia self-replenish in the brain and spinal cord throughout life, independent of circulating monocyte precursors, presumably by a subset of radio-resistant precursors derived from the yolk sac $[31,59]$. Despite the new data, the repopulation dynamics of CNS microglia will undoubtedly remain a controversial area of research. Indeed, circulating monocytes do enter the pathological CNS where they can differentiate into cells that are phenotypically and morphologically indistinguishable from resident microglia $[60,61]$. Whether these former cells possess molecular and functional characteristics that are distinct from resident microglia is not known.

Neutrophils and monocytes originate from a common precursor (in bone marrow), and then travel through the blood to the site of the injury in response to CXC and CC chemokines, respectively (FIG. 2). Chemokines are produced within and outside the spinal cord. After injury to the brain or spinal cord, resident tissue macrophages in liver (Kuppfer cells [KCs]) are pivotal in regulating mobilization of leukocytes to the site of injury. KCs produce CXC ligand-10 (CXCL-10), IL-1 $\beta$, CC ligand-2 (CCL-2), and macrophage inflammatory protein (MIP$1 \alpha)$ [62]. When KCs are depleted via systemic injection of clodronate liposomes, accumulation of neutrophils in the injured brain or spinal cord is significantly reduced [63]. This may partially explain why post-injury intravenous delivery of clodronate liposomes confers neuroprotection and promotes recovery of function after SCI $[26,64,65]$.

For monocytes, emigration from the bone marrow is chemokine receptor $\mathrm{CC}$ receptor 2 (CCR2)-dependent $[66,67]$. Recent data show that monocyte recruitment and survival also are CX3C ligand-1 (CX3CL1)/CX3C receptor-1 (CX3CR1)-dependent [68]. After infection with the intracellular bacterium Listeria monocytogenes, classical "inflammatory" Ly-6C ${ }^{\text {high }}$ monocytes are maintained in the bone marrow of $\mathrm{CCR} 2^{-/-}$mice [67]. After SCI, genetic deletion of CCR2 impairs monocyte infiltration and phagocytic uptake of degenerating myelin but does not permanently abrogate intraspinal macrophage accumulation [69]. It is likely that in the absence of CCR2+ monocytes there is compensatory proliferation of resident microglia and perhaps increased recruitment of $\mathrm{Ly} 6 \mathrm{C}^{\mathrm{low}} / \mathrm{CCR} 2^{\text {low }} / \mathrm{CX} 3 \mathrm{CR} 1^{\text {high }}$ monocyte subsets; however, the relative contribution of different monocyte subsets to neuroinflammatory processes after SCI has not been determined (see as follows).

Although it is believed that most circulating leukocytes are derived from bone marrow, recent data indicate that the spleen may rapidly deploy a large population of monocytes into the circulation in response to ischemic injury [70] (FIG. 2). In a model of myocardial ischemia, splenectomy markedly reduced intracardiac monocyte accumulation [70]. Importantly, splenectomy did not elicit a compensatory release of CCR2 + monocytes from the bone marrow. The splenic reservoir also has been shown to be important in regulating tissue injury in a model of cerebral ischemia. Splenectomy 2 weeks prior to a middle cerebral artery occlusion was associated with a significant reduction in monocyte accumulation and lesion size in the ischemic brain [71].

The mechanisms regulating leukocyte mobilization from the spleen after injury may be numerous. After middle cerebral artery occlusion, catecholamines are released into the blood and may activate adrenergic receptors in the spleen causing the release of monocytes [72]. In the myocardial infarction model, splenic release of monocytes was impaired in angiotensin $\mathrm{II}^{-/-}$mice [70]. Whether splenic monocytes contribute to the intraspinal macrophage response elicited by traumatic SCI, in which ischemia is only one component of the pathophysiology, has not been studied. 


\section{Mechanisms regulating intraspinal accumulation of myeloid cells after SCI}

After release from the bone marrow (or spleen), leukocyte transmigration from blood to spinal cord is dependent on a cadre of adhesion molecules and chemokines [73-75]. Details of the cellular and molecular characteristics of the multi-step paradigm for leukocyteendothelial interactions at the blood-brain barrier have been published elsewhere [73-75]. A more comprehensive review of how the complex interplay between neurons and glia regulates the onset of neuroinflammatory cascades after SCI, including the recruitment of neutrophils and monocytes, can be found in recent reviews from our laboratory $[3,5]$. Here we highlight newer data generated in rat and mouse models of SCI that reveal novel control mechanisms for myeloid cell entry and activation after SCI.

Within minutes to hours post-injury, intraspinal neurons and astrocytes produce IL-1 $\beta$ [76, 77]. Synthesis of bioactive IL-1 $\beta$ in neurons is dependent on the NALP1 inflammasome [76]. A similar mechanism is likely in astrocytes, microglia, and oligodendrocytes, although this has not been proven after SCI [78]. In astrocytes, the synthesis of IL-1 $\beta$ and chemokines (e.g., CCL2, CCL3/ MIP- $1 \alpha$, CXCL1/KC, and CXCL2/MIP-2) is triggered via the IL-1 receptor and not by pattern recognition receptors (TLR2, TLR4) [79]. In the absence of MyD88/IL-1receptor-dependent signaling, entry of neutrophils and to a lesser extent monocytes are reduced after SCI [79].

Myeloid cell entry into the injured spinal cord and associated changes in vascular permeability also require the induction of matrix metalloproteinases (MMPs) (see review in this issue [80] and also see Noble et al. [81] and Wells et al. [82]). In rat and human myeloid cells, CD95 ligand (CD95L)/CD95 interactions are pivotal for induction of MMP-9 and subsequent infiltration into the injured spinal cord [83]. When CD95L is deleted from myeloid cells, intraspinal inflammation is reduced and recovery of function is improved after experimental SCI [83]. Thus, chemotactic gradients at the injury site are established primarily by nonmyeloid cells, and then later, as cells begin to enter the injury site, myeloid cells amplify this response. In fact, the sequential recruitment and activation of neutrophils and monocytes is essential for amplifying and then resolving inflammatory cascades [84]. However, after SCI, cellular and molecular indices of neuroinflammation exist chronically throughout the injured spinal cord, suggesting that the resolution of inflammation is impaired. Currently, there is no explanation for this effect, and further research is needed.

\section{Characterizing myeloid cell heterogeneity in the injured spinal cord}

Spinal cord injury elicits and activates a heterogeneous population of myeloid cells (e.g., neutrophils, microglia, and monocytes) with the ability to exacerbate tissue injury and promote repair. This phenotypic and functional heterogeneity is maintained by diverse signaling cues in injured and spared tissues. For example, neuroimmune regulatory proteins present on neurons and glia regulate the phenotype and function of microglia. Neuroimmune regulatory proteins include CD47, CD22, CD95L, CD200 and CX3CL1 [5]. Loss or downregulation of these ligands causes microglia and monocytederived macrophages to become hyperreactive and potentially destructive $[5,85,86]$. CD200/CD200R interactions are especially intriguing because CD200R is expressed only on myeloid cells.

Intrinsic heterogeneity within the pool of resident and recruited myeloid cells results, in part, from differences in cell maturation, differentiation, and activation. A variety of antibodies are used to characterize these diverse cell subsets; however, the expression profiles of surface and cytoplasmic antigens often overlap, making it difficult to assign a definitive phenotype or function to a given cell type. For example, Ly6G/Gr-1 is widely used as specific marker for neutrophils; however, it also labels a subset of monocytes [87]. Therefore, when used as a purported neutrophil-depleting antibody, the effects of Ly6G/Gr-1-mediated cell depletion may also result from loss of monocytes. Also, granulocytic MDSCs express Ly6G and CD11b, but are distinct from monocytic MDSCs, which express CD11b and Ly6C [29]. These phenotypic distinctions are important to consider when interpreting data in which antibodies are used for depletion of circulating leukocyte subsets. For example, the Ly6G/Gr-1 antibody was used to deplete neutrophils in a mouse model of SCI [23]. The antibody was injected systemically at 2 and $24 \mathrm{~h}$ post-SCI, and was found to significantly reduce the number of circulating neutrophils (but not monocytes) and inflammatory cytokines, but lesion pathology and locomotor recovery were impaired in Gr-1-treated mice. From these data, one can conclude that neutrophils are important for limiting lesion pathology after SCI and should not be a target for depletion. However, future studies should also explore whether Gr-1 antibodies deplete granulocytic MDSCs. These cells have not been evaluated after SCI, but may play an important yet unappreciated role in regulating acute post-traumatic inflammation.

Flow cytometry can be used to distinguish between these closely related cell types, although one loses the ability to co-localize cells to areas of injury or repair in the spinal cord. Using flow cytometry myeloid cell subsets can be defined, based on physical properties (e.g., cell size, granularity) and expression of cell membrane or cytoplasmic antigens. For example, blood monocytes stain positive for CD11b and M-CSF receptors (CSF-1R or CD115) [88]. Neutrophils also express $\mathrm{CD} 11 \mathrm{~b}$, but are distinguished from blood monocytes by 
Table 1. Pre-Clinical Manipulation of Myeloid Cells after Experimental Spinal Cord Injury

\begin{tabular}{|c|c|c|}
\hline Putative Target & Approach & Outcome [Reference No.] \\
\hline \multirow[t]{4}{*}{$\begin{array}{l}\text { Monocytes/ } \\
\text { macrophages }\end{array}$} & $\begin{array}{l}\text { Post-injury (intravenous) } \\
\text { injections of clodronate liposomes }\end{array}$ & $\begin{array}{l}\text { Reduced macrophages/microglia in the spinal cord; } \\
\text { improved axon and myelin sparing; increased segmental } \\
\text { axonal sprouting; improved gross locomotor function [65] }\end{array}$ \\
\hline & Clodronate liposomes + rolipram & $\begin{array}{l}\text { Additive neuroprotection and improvements in spontaneous } \\
\text { neurological recovery [64] }\end{array}$ \\
\hline & Colchicine + chloroquine & $\begin{array}{l}\text { Attenuated intraspinal inflammation; neuroprotection with } \\
\text { improved motor and bladder function }[98,99]\end{array}$ \\
\hline & Intraperitoneal silica dust & $\begin{array}{l}\text { Delayed secondary loss of function (guinea pig SCI model); } \\
\text { reduced demyelination and hypervascularity }[97,98]\end{array}$ \\
\hline $\begin{array}{l}\text { Microglia } \\
(+ \text { macrophages })\end{array}$ & Minocycline & $\begin{array}{l}\text { Reduced lesion size; axonal sparing; functional } \\
\text { improvement [96] }\end{array}$ \\
\hline \multirow[t]{2}{*}{ Neutrophils } & Activated protein $\mathrm{C}$ (APC) & $\begin{array}{l}\text { Inhibited neutrophil activation in model of ischemic SCI; } \\
\text { attenuated petechial hemorrhage and neurological deficits [49] }\end{array}$ \\
\hline & Inject nitrogen mustard & $\begin{array}{l}\text { Decreased intraspinal neutrophils; improved recovery of } \\
\text { motor function }[100,101]\end{array}$ \\
\hline $\begin{array}{l}\text { Macrophages/microglia } \\
\text { and neutrophils }\end{array}$ & Anti-CD11d antibodies & $\begin{array}{l}\text { Improved recovery of motor, sensory and autonomic functions; } \\
\text { neuroprotection; reduced oxidative stress, intraspinal leukocyte } \\
\text { accumulation and proinflammatory cytokine expression } \\
{[92-95,105]}\end{array}$ \\
\hline \multirow[t]{4}{*}{$\begin{array}{l}\text { Lesion environment; } \\
\text { cytokine milieu }\end{array}$} & Anti-IL-6 receptor antibodies & $\begin{array}{l}\text { Reduced monocyte-derived macrophages in spinal cord; } \\
\text { enhanced microglial response to SCI; improved recovery } \\
\text { of function }[44,52]\end{array}$ \\
\hline & Inhibit leukotriene B4 (LTB4) & $\begin{array}{l}\text { Reduced intraspinal leukocytes and inflammatory cytokines; } \\
\text { neuroprotection; improved functional recovery }[44,52]\end{array}$ \\
\hline & Infusion of GM-CSF & $\begin{array}{l}\text { Increased intraspinal BDNF; enhanced neural progenitor } \\
\text { cell turnover; improved acute recovery of locomotor function } \\
{[28,110]}\end{array}$ \\
\hline & IL-10 & $\begin{array}{l}\text { Reduced intraspinal inflammation; neuroprotection; } \\
\text { improved recovery of locomotor function [106-108] }\end{array}$ \\
\hline
\end{tabular}

$\mathrm{BDNF}=$ brain-derived neurotrophic factor; $\mathrm{GM}-\mathrm{CSF}=$ granulocyte-macrophage colony stimulating factor; $\mathrm{IL}=$ interleukin; $\mathrm{SCI}=$ spinal cord injury.

their relative expression of CD45, Gr-1, and F4/80 [89]. Neutrophils are $\mathrm{CD} 45+/ \mathrm{Gr}^{+} 1^{+} / \mathrm{F} 4 / 80^{-}$, whereas monocytes are $\mathrm{CD} 45^{+} / \mathrm{Gr}-1^{-} / \mathrm{F} 4 / 80^{+}$[89]. Relative levels of Ly6C can be used to differentiate between mature and immature monocytes/macrophages; high Ly6C levels are associated with immature or younger monocyte precursors ("inflammatory" monocytes) and low levels with a more mature cell type that has reduced migratory capacity (i.e., "resident or noninflammatory" monocytes) [88]. The relative expression of CD45 can also be used to distinguish between CNS macrophages that are derived from microglia $\left(\mathrm{CD} 45^{\text {low }}\right)$ and blood monocytes (CD45 $\left.{ }^{\text {high }}\right)$ [89].

The previously described parameters are useful for broadly categorizing phenotypic and functional heterogeneity of myeloid cells at and nearby the site of injury. However, it is likely that as the lesion evolves, signaling moieties in the lesion microenvironment will also change, causing functional adaptivity in myeloid cells, especially CNS macrophages $[5,90]$. Recent work from our laboratory has shown that SCI elicits a diverse macrophage response that changes as a function of time post-injury in mice. Early after injury (for at least 3 days), the lesion site is comprised of macrophages that can be defined as "classically activated" pro- inflammatory M1 cells and "alternatively activated" M2 cells. M1 cells express discrete phenotypic markers including inducible nitric oxide synthase (iNOS), CD86, and CD16/32, whereas M2 cells express CD206 and arginase I [24]. After $\sim 7$ days, the ratio of M1:M2 cells increases due to a concomitant increase in expression of M1 cell markers and a decrease in cells with an M2 phenotype. The significance of this is not clear; however, M1 macrophages are neurotoxic and promote dieback of injured CNS axons [24-26], whereas M2 macrophages seem to have no adverse effect on neurons and can promote growth of axons across inhibitory substrates that dominate sites of injury [24]. In an independent study, a subset of IL-10+ monocyte-derived macrophages was found to be anti-inflammatory and essential for tissue repair [91]. Whether CNS macrophage heterogeneity can be controlled for therapeutic gain after SCI, either through direct manipulation of myeloid cell subsets and/or the lesion environment, awaits further research.

\section{Experimental therapies}

Various pharmacological agents, toxins and antibodies have been used to deplete or functionally inactivate 
neutrophils, microglia, and/or monocyte-derived macrophages in pre-clinical models of SCI. A partial list of these pre-clinical therapies is summarized in Table 1. These various experiments, including the use of clodronate liposomes [64, 65], anti-CD11d antibodies [92-95], minocycline [96], silica dust $[1,97,98]$, colchicine and chloroquine [98, 99], activated protein $\mathrm{C}$ [49], and nitrogen mustard $[100,101]$ have been reviewed previously $[1,5,98]$ and will not be discussed further. Additionally, because 2 other review articles in this issue provide an overview of the use of MMP inhibitors for SCI [80] and provide new strategies to manipulate microglia (and macrophage) metabotropic glutamate receptors [102], we will not discuss these topics any further. Instead, we will focus on newer approaches in which blockade or infusion of select cytokines has been shown to modify the composition and phenotype of the responding myeloid cells. Additionally, the implications of recent Gr1-mediated neutrophil depletion data are described in the context of myeloid cell heterogeneity and downstream wound healing cascades.

IL-6 is a pro-inflammatory cytokine that promotes the differentiation of monocytes into macrophages [92-95] and can also elicit monocyte recruitment [103]. When over-expressed in the injured spinal cord using genetically modified cellular vectors, excess IL- 6 enhanced the endogenous neutrophil and monocyte response to SCI and exacerbated lesion pathology [104]. In mice, postinjury intraperitoneal injection of MR16-1, an antibody specific for the IL-6 receptor, profoundly affected intraspinal inflammation, lesion pathology, and functional recovery [44, 52]. Indeed, a single injection of MR16-1 reduced intraspinal inflammation and pro-inflammatory chemokine/cytokine signaling. Interestingly, MR16-1 also changed the composition of the responding myeloid cells. A single injection shifted the normal cell dynamics, resulting in a microglial-dominant reaction. At chronic times post-injury, microglial-dominant inflammation was associated with increased axonal sparing distal to and at the site of injury, and improved functional recovery. These data show that rather than deplete cells, the destructive potential of myeloid cells can be reduced (or abolished) by interfering with select acute inflammatory signaling cascades early after injury.

A similar result was achieved in SCI by blocking leukotriene B4 (LTB4) signaling. LTB4 is a lipid chemoattractant produced from membrane phospholipids as part of the arachidonic acid cascade. LTB4 binds to LTB4 receptor 1 (BLT1) on neutrophils and monocytes, enhancing their recruitment and activation at sites of inflammation [103]. After SCI, inflammatory cascades are significantly reduced in $\mathrm{BLT1}^{-/-}$mice and in $\mathrm{SCI}$ wild-type mice treated with the LTB4 receptor antagonist, ONO-4057 [105]. Indeed, infiltration of neutrophils and monocytes/macrophages was reduced in parallel with various myeloid-specific cytokines and chemokines, including IL-6, IL-1 $\beta$, TNF- $\alpha$, CXCL1, CXL2, and CCL2. These changes were associated with decreased neuronal apoptosis, enhanced conduction in axons traversing the site of injury, and improved locomotor recovery at chronic post-injury survival periods.

In contrast to blocking pro-inflammatory signaling, it may be possible to boost local concentrations of antiinflammatory cytokines. For example, local infusions of IL10 are neuroprotective after SCI, in part because the cytokine can antagonize pro-inflammatory signaling (e.g., NF-kB) at the site of injury [106-108]. IL-4 is another anti-inflammatory cytokine that may be important for regulating intraspinal inflammation. After SCI, neutrophils transiently produce IL-4 [109]; however, the eventual polarization of the CNS macrophage response toward a neurotoxic M1 phenotype suggests that endogenous IL-4dependent immune regulation is inefficient. Clearly, some intrinsic immune regulation is mediated by IL-4 because injection of anti-IL-4 antibodies after SCI exacerbates proinflammatory signaling [109]. Blockade of endogenous IL4 leads to an increase in CCL2 synthesis and myeloid cell influx to the injury site with a concomitant increase in lesion pathology [109]. Whether focal and sustained infusion of IL-4 could alleviate acute macrophage-mediated toxicity is under investigation. Still, caution must be exercised with cytokine therapies, especially because cytokines are pleiotropic and can have broad "off-target" effects. For example, granulocyte-macrophage colony stimulating factor is beneficial after SCI; however, its mechanism of action is difficult to pinpoint. GM-CSF exerts anti-apoptotic effects on neurons [51, 110, 111]; however, recent data indicate that GM-CSF infusion increases intraspinal brain-derived neurotrophic factor and improves locomotor recovery via mechanisms involving the activation of dendritic cells and neural stem/progenitor cells [28]. Mice treated with GMCSF also had more brain-derived neurotrophic factor production and better locomotor recovery.

\section{CONCLUSIONS}

Spinal cord injury elicits an inflammatory response comprised of both resident and newly recruited peripheral myeloid cells. These cells can positively and negatively affect the spinal cord and tissues in the periphery. Although the bone marrow is widely believed to be the primary source of circulating myeloid cells after SCI, recent data implicate the spleen as an emergency reservoir for monocytes. Whether the spleen contributes to intraspinal inflammation has not been determined. As myeloid cells accumulate at the site of injury, their impact on spinal cord structure and function will be determined by their phenotype and the immune-modulatory signals that exist within the lesion environment. 
The precise nature of these signals and how they regulate macrophage phenotype and function are unknown and more research is needed to develop therapies that effectively target select myeloid cell subsets and/or factors that regulate myeloid cell differentiation and function. New studies show that it may be possible to infuse select cytokines or cytokine receptor antagonists to alter the composition and dynamics of myeloid cells. Given the early and sustained presence of myeloid cells at and nearby sites of SCI, these cells represent ideal therapeutic targets for minimizing secondary tissue injury and/or promoting repair of the injured spinal cord.

Acknowledgment: Full conflict of interest disclosure is available in the electronic supplementary material for this article.

\section{REFERENCES}

1. Alexander JK, Popovich PG. Neuroinflammation in spinal cord injury: therapeutic targets for neuroprotection and regeneration. Prog Brain Res 2009;175:125-137.

2. Ankeny DP, Popovich PG. B cells and autoantibodies: complex roles in CNS injury. Trends Immunol 2010;31:332-338.

3. Donnelly DJ, Popovich PG. Inflammation and its role in neuroprotection, axonal regeneration and functional recovery after spinal cord injury. Exp Neurol 2008;209:378-388.

4. Popovich P, McTigue D. Damage control in the nervous system: beware the immune system in spinal cord injury. Nat Med 2009;15:736-737.

5. Popovich PG, Longbrake EE. Can the immune system be harnessed to repair the CNS? Nat Rev Neurosci 2008;9:481-493.

6. Popovich PG, Hickey WF. Bone marrow chimeric rats reveal the unique distribution of resident and recruited macrophages in the contused rat spinal cord. J Neuropathol Exp Neurol 2001;60:676-685.

7. Ransohoff RM, Cardona AE. The myeloid cells of the central nervous system parenchyma. Nature 2010;468:253-262.

8. Dibaj P, Nadrigny F, Steffens H, et al. NO mediates microglial response to acute spinal cord injury under ATP control in vivo. Glia 2010;58:1133-1144.

9. Beck KD, Nguyen HX, Galvan MD, Salazar DL, Woodruff TM, Anderson AJ. Quantitative analysis of cellular inflammation after traumatic spinal cord injury: evidence for a multiphasic inflammatory response in the acute to chronic environment. Brain 2010;133 (Pt 2):433-447.

10. Saiwai H, Ohkawa Y, Yamada $H$ et al. The LTB4-BLT1 axis mediates neutrophil infiltration and secondary injury in experimental spinal cord injury. Am J Pathol 2010;176:2352-2366.

11. Stirling DP, Yong VW. Dynamics of the inflammatory response after murine spinal cord injury revealed by flow cytometry. J Neurosci Res 2008;86:1944-1958.

12. Sroga JM, Jones TB, Kigerl KA, McGaughy VM, Popovich PG. Rats and mice exhibit distinct inflammatory reactions after spinal cord injury. J Comp Neurol 2003;462:223-240.

13. Zhu B, Bando Y, Xiao S, et al. CD11b + Ly-6 C(hi) suppressive monocytes in experimental autoimmune encephalomyelitis. $\mathrm{J}$ Immunol 2007;179:5228-5237.

14. Popovich PG, Wei P, Stokes BT. Cellular inflammatory response after spinal cord injury in Sprague-Dawley and Lewis rats. J Comp Neurol 1997;377:443-464.

15. Blight AR. Morphometric analysis of a model of spinal cord injury in guinea pigs, with behavioral evidence of delayed secondary pathology. J Neurol Sci 1991:103:156-171.

16. Blight AR. Cellular morphology of chronic spinal cord injury in the cat: analysis of myelinated axons by line-sampling. Neuroscience 1982;10:521-543.
17. Kigerl KA, McGaughy VM, Popovich PG. Comparative analysis of lesion development and intraspinal inflammation in four strains of mice following spinal contusion injury. J Comp Neurol 2006;494:578-594.

18. Fleming JC, Norenberg MD, Ramsay DA, et al. The cellular inflammatory response in human spinal cords after injury. Brain 2006;129(Pt 12):3249-3269.

19. Pillay J, den Braber I, Vrisekoop N, et al. In vivo labeling with $2 \mathrm{H} 2 \mathrm{O}$ reveals a human neutrophil lifespan of 5.4 days. Blood 2010;116:625-627.

20. Murphy J, Summer R, Wilson AA, Kotton DN, Fine A. The prolonged life-span of alveolar macrophages. Am J Respir Cell Mol Biol 2008;38:380-385.

21. Taoka Y, Okajima K. Role of leukocytes in spinal cord injury in rats. J Neurotrauma 2000;17:219-229.

22. Taoka Y, Okajima K, Murakami K, Johno M, Naruo M. Role of neutrophil elastase in compression-induced spinal cord injury in rats. Brain Res 1998;799:264-269.

23. Stirling DP, Liu S, Kubes P, Yong VW. Depletion of Ly6G/Gr-1 leukocytes after spinal cord injury in mice alters wound healing and worsens neurological outcome. J Neurosci 2009;29:753-764.

24. Kigerl KA, Gensel JC, Ankeny DP, Alexander JK, Donnelly DJ, Popovich PG. Identification of two distinct macrophage subsets with divergent effects causing either neurotoxicity or regeneration in the injured mouse spinal cord. J Neurosci 2009;29:1343513444.

25. Busch SA, Horn KP, Silver DJ, Silver J. Overcoming macrophagemediated axonal dieback following CNS injury. J Neurosci 2009;29:9967-9976.

26. Horn KP, Busch SA, Hawthorne AL, van Rooijen N, Silver J. Another barrier to regeneration in the CNS: activated macrophages induce extensive retraction of dystrophic axons through direct physical interactions. J Neurosci 2008;28:9330-9341.

27. Yaguchi M, Tabuse M, Ohta S, et al. Transplantation of dendritic cells promotes functional recovery from spinal cord injury in common marmoset. Neurosci Res 2009;65:384-392.

28. Hayashi K, Ohta S, Kawakami Y, Toda M. Activation of dendriticlike cells and neural stem/progenitor cells in injured spinal cord by GM-CSF. Neurosci Res 2009;64:96-103.

29. Gabrilovich DI, Nagaraj S. Myeloid-derived suppressor cells as regulators of the immune system. Nat Rev Immunol 2009;9:162174.

30. Bao F, Bailey CS, Gurr KR, et al. Increased oxidative activity in human blood neutrophils and monocytes after spinal cord injury. Exp Neurol 2009;215:308-316.

31. Ginhoux F, Greter M, Leboeuf M, et al. Fate mapping analysis reveals that adult microglia derive from primitive macrophages. Science 2010;330:841-845.

32. Orkin SH, Zon LI. Hematopoiesis: an evolving paradigm for stem cell biology. Cell 2008;132:631-644.

33. Bell JJ, Bhandoola A. The earliest thymic progenitors for T cells possess myeloid lineage potential. Nature 2008;452:764-767.

34. Bhandoola A, von Boehmer H, Petrie HT, Zuniga-Pflucker JC. Commitment and developmental potential of extrathymic and intrathymic $\mathrm{T}$ cell precursors: plenty to choose from. Immunity 2007;26:678-689.

35. Katsura Y. Redefinition of lymphoid progenitors. Nat Rev Immunol 2002;2:127-132.

36. Lu M, Kawamoto H, Katsube Y, Ikawa T, Katsura Y. The common myelolymphoid progenitor: a key intermediate stage in hemopoiesis generating T and B cells. J Immunol 2002;169:3519-3525.

37. Wada H, Masuda K, Satoh R, et al. Adult T-cell progenitors retain myeloid potential. Nature 2008;452:768-772.

38. Welner RS, Pelayo R, Kincade PW. Evolving views on the genealogy of B cells. Nat Rev Immunol 2008;8:95-106.

39. Doulatov S, Notta F, Eppert K, Nguyen LT, Ohashi PS, Dick JE. Revised map of the human progenitor hierarchy shows the origin of macrophages and dendritic cells in early lymphoid development. Nat Immunol 2010;11:585-593.

40. Dorshkind K. Not a split decision for human hematopoiesis. Nat Immunol 2010;11:569-570.

41. Aiello FB, Keller JR, Klarmann KD, Dranoff G, Mazzucchelli R, Durum SK. IL-7 induces myelopoiesis and erythropoiesis. J Immunol 2007;178:1553-1563. 
42. Barreda DR, Hanington PC, Belosevic M. Regulation of myeloid development and function by colony stimulating factors. Dev Comp Immunol 2004;28:509-554.

43. Du X, Williams DA. Interleukin-11: review of molecular, cell biology, and clinical use. Blood 1997;89:3897-3908.

44. Mukaino M, Nakamura M, Yamada O, et al. Anti-IL-6-receptor antibody promotes repair of spinal cord injury by inducing microglia-dominant inflammation. Exp Neurol 2010;224:403414.

45. Shurin MR, Esche C, Lotze MT. FLT3: receptor and ligand. Biology and potential clinical application. Cytokine Growth Factor Rev 1998;9:37-48.

46. Damia G, Komschlies KL, Faltynek CR, Ruscetti FW, Wiltrout RH. Administration of recombinant human interleukin-7 alters the frequency and number of myeloid progenitor cells in the bone marrow and spleen of mice. Blood 1992;79:1121-1129.

47. Faltynek CR, Wang S, Miller D, et al. Administration of human recombinant IL-7 to normal and irradiated mice increases the numbers of lymphocytes and some immature cells of the myeloid lineage. J Immunol 1992;149:1276-1282.

48. Grzegorzewski KJ, Komschlies KL, Jacobsen SE, Ruscetti FW, Keller JR, Wiltrout RH. Mobilization of long-term reconstituting hematopoietic stem cells in mice by recombinant human interleukin 7. J Exp Med 1995;181:369-374.

49. Hirose K, Okajima K, Taoka Y, et al. Activated protein C reduces the ischemia/reperfusion-induced spinal cord injury in rats by inhibiting neutrophil activation. Ann Surg 2000;232:272-280.

50. Bartholdi D, Schwab ME. Expression of pro-inflammatory cytokine and chemokine mRNA upon experimental spinal cord injury in mouse: an in situ hybridization study. Eur J Neurosci 1997;9:1422-1438.

51. Ousman SS, David S. MIP-1alpha, MCP-1, GM-CSF, and TNFalpha control the immune cell response that mediates rapid phagocytosis of myelin from the adult mouse spinal cord. J Neurosci 2001;21:4649-4656.

52. Okada S, Nakamura M, Mikami Y, et al. Blockade of interleukin-6 receptor suppresses reactive astrogliosis and ameliorates functional recovery in experimental spinal cord injury. J Neurosci Res 2004;76:265-276.

53. Streit WJ, Semple-Rowland SL, Hurley SD, Miller RC, Popovich PG, Stokes BT. Cytokine mRNA profiles in contused spinal cord and axotomized facial nucleus suggest a beneficial role for inflammation and gliosis. Exp Neurol 1998;152:74-87.

54. Lee YL, Shih K, Bao P, Ghirnikar RS, Eng LF. Cytokine chemokine expression in contused rat spinal cord. Neurochem Int 2000;36:417-425.

55. Kwon BK, Stammers AM, Belanger LM, et al. Cerebrospinal fluid inflammatory cytokines and biomarkers of injury severity in acute human spinal cord injury. J Neurotrauma 2010;27:669-682.

56. Chan WY, Kohsaka S, Rezaie P. The origin and cell lineage of microglia: new concepts. Brain Res Rev 2007;53:344-354.

57. Perry VH, Hume DA, Gordon S. Immunohistochemical localization of macrophages and microglia in the adult and developing mouse brain. Neuroscience 1985;15:313-326.

58. Rezaie P, Male D. Mesoglia \& microglia-a historical review of the concept of mononuclear phagocytes within the central nervous system. J Hist Neurosci 2002;11:325-374.

59. Ajami B, Bennett JL, Krieger C, Tetzlaff W, Rossi FM. Local selfrenewal can sustain CNS microglia maintenance and function throughout adult life. Nat Neurosci 2007;10:1538-1543.

60. Krall WJ, Challita PM, Perlmutter LS, Skelton DC, Kohn DB. Cells expressing human glucocerebrosidase from a retroviral vector repopulate macrophages and central nervous system microglia after murine bone marrow transplantation. Blood 1994;83:2737-2748.

61. Lewis CA, Solomon JN, Rossi FM, Krieger C. Bone marrowderived cells in the central nervous system of a mouse model of amyotrophic lateral sclerosis are associated with blood vessels and express CX(3)CR1. Glia 2009;57:1410-1419.

62. Campbell SJ, Perry VH, Pitossi FJ, et al. Central nervous system injury triggers hepatic $\mathrm{CC}$ and $\mathrm{CXC}$ chemokine expression that is associated with leukocyte mobilization and recruitment to both the central nervous system and the liver. Am J Pathol 2005;166:14871497.
63. Campbell SJ, Zahid I, Losey P, et al. Liver Kupffer cells control the magnitude of the inflammatory response in the injured brain and spinal cord. Neuropharmacology 2008;55:780-787.

64. Iannotti CA, Clark M, Horn KP, van Rooijen N, Silver J, Steinmetz MP. A combination immunomodulatory treatment promotes neuroprotection and locomotor recovery after contusion SCI. Exp Neurol 2010. doi:10.1016/j.expneurol.2010.03.010.

65. Popovich PG, Guan Z, Wei P, Huitinga I, van Rooijen N, Stokes BT. Depletion of hematogenous macrophages promotes partial hindlimb recovery and neuroanatomical repair after experimental spinal cord injury. Exp Neurol 1999;158:351-365.

66. Crane MJ, Hokeness-Antonelli KL, Salazar-Mather TP. Regulation of inflammatory monocyte/macrophage recruitment from the bone marrow during murine cytomegalovirus infection: role for type I interferons in localized induction of CCR2 ligands. J Immunol 2009; 183:2810-2817.

67. Serbina NV, Pamer EG. Monocyte emigration from bone marrow during bacterial infection requires signals mediated by chemokine receptor CCR2. Nat Immunol 2006;7:311-317.

68. Landsman L, Bar-On L, Zernecke A, et al. CX3CR1 is required for monocyte homeostasis and atherogenesis by promoting cell survival. Blood 2009;113:963-972.

69. Ma M, Wei T, Boring L, Charo IF, Ransohoff RM, Jakeman LB. Monocyte recruitment and myelin removal are delayed following spinal cord injury in mice with CCR2 chemokine receptor deletion. J Neurosci Res 2002;68:691-702.

70. Swirski FK, Nahrendorf M, Etzrodt M, et al. Identification of splenic reservoir monocytes and their deployment to inflammatory sites. Science 2009;325:612-616.

71. Ajmo CT Jr., Vernon DO, Collier L, et al. The spleen contributes to stroke-induced neurodegeneration. J Neurosci Res 2008;86:2227-2234.

72. Ajmo CT Jr., Collier LA, Leonardo CC, et al. Blockade of adrenoreceptors inhibits the splenic response to stroke. Exp Neurol 2009;218:47-55.

73. Greenwood J, Heasman SJ, Alvarez JI, Prat A, Lyck R, Engelhardt B. Leukocyte-endothelial cell crosstalk at the blood-brain barrier: a prerequisite for successful immune cell entry to the brain. Neuropathol Appl Neurobiol 2011;37:24-39.

74. Man S, Ubogu EE, Ransohoff RM. Inflammatory cell migration into the central nervous system: a few new twists on an old tale. Brain Pathol 2007; 17:243-250.

75. Imhof BA, Aurrand-Lions M. Adhesion mechanisms regulating the migration of monocytes. Nat Rev Immunol 2004;4:432-444.

76. de Rivero Vaccari JP, Lotocki G, Marcillo AE, Dietrich WD, Keane RW. A molecular platform in neurons regulates inflammation after spinal cord injury. J Neurosci 2008; 28:3404-3414.

77. Pineau I, Lacroix S. Proinflammatory cytokine synthesis in the injured mouse spinal cord: multiphasic expression pattern and identification of the cell types involved. J Comp Neurol 2007;500:267-285.

78. Loane DJ, Byrnes KR. Role of microglia in neurotrauma. Neurotherapeutics 2010;7:366-377.

79. Pineau I, Sun L, Bastien D, Lacroix S. Astrocytes initiate inflammation in the injured mouse spinal cord by promoting the entry of neutrophils and inflammatory monocytes in an IL-1 receptor/MyD88-dependent fashion. Brain Behav Immun 2010;24:540-553.

80. Zhang H, Chang M, Hansen CN, Basso DM, Noble-Haeusslein LJ. Role of matrix metalloproteinases and therapeutic benefits of their inhibition in spinal cord injury. Neurotherapeutics. doi:10.1007/ s13311-011-0038-0.

81. Noble LJ, Donovan F, Igarashi T, Goussev S, Werb Z. Matrix metalloproteinases limit functional recovery after spinal cord injury by modulation of early vascular events. J Neurosci 2002;22:7526-7535.

82. Wells JE, Rice TK, Nuttall RK, et al. An adverse role for matrix metalloproteinase 12 after spinal cord injury in mice. J Neurosci 2003;23:10107-10115.

83. Letellier E, Kumar S, Sancho-Martinez I, et al. CD95-ligand on peripheral myeloid cells activates Syk kinase to trigger their recruitment to the inflammatory site. Immunity 2010; 32:240-252.

84. Soehnlein O, Lindbom L. Phagocyte partnership during the onset and resolution of inflammation. Nat Rev Immunol 2010;10:427-439.

85. Hokeness-Antonelli KL, Crane MJ, Dragoi AM, Chu WM, SalazarMather TP. IFN-alphabeta-mediated inflammatory responses and 
antiviral defense in liver is TLR9-independent but MyD88-dependent during murine cytomegalovirus infection. J Immunol 2007;179:6176-6183.

86. Neumann H. Control of glial immune function by neurons. Glia 2001;36:191-199.

87. Nagendra S, Schlueter AJ. Absence of cross-reactivity between murine Ly-6 C and Ly-6 G. Cytometry A 2004;58:195-200.

88. Sunderkotter C, Nikolic T, Dillon MJ, et al. Subpopulations of mouse blood monocytes differ in maturation stage and inflammatory response. J Immunol 2004;172:4410-4417.

89. Byrnes KR, Loane DJ, Faden AI. Metabotropic glutamate receptors as targets for multipotential treatment of neurological disorders. Neurotherapeutics 2009;6:94-107.

90. Suttles J, Stout RD. Macrophage CD40 signaling: a pivotal regulator of disease protection and pathogenesis. Semin Immunol 2009;21:257-264.

91. Shechter R, London A, Varol C, et al. Infiltrating blood-derived macrophages are vital cells playing an anti-inflammatory role in recovery from spinal cord injury in mice. PLoS Med 2009;6:e1000113.

92. Saville LR, Pospisil CH, Mawhinney LA, et al. A monoclonal antibody to CD11d reduces the inflammatory infiltrate into the injured spinal cord: a potential neuroprotective treatment. J Neuroimmunol 2004;156:42-57.

93. Gris D, Marsh DR, Oatway MA, et al. Transient blockade of the CD11d/CD18 integrin reduces secondary damage after spinal cord injury, improving sensory, autonomic, and motor function. J Neurosci 2004;24:4043-4051.

94. Bao F, Dekaban GA, Weaver LC. Anti-CD11d antibody treatment reduces free radical formation and cell death in the injured spinal cord of rats. J Neurochem 2005;94:1361-1373.

95. Gris P, Tighe A, Thawer S, et al. Gene expression profiling in antiCD11d mAb-treated spinal cord-injured rats. J Neuroimmunol 2009;209:104-113.

96. Wells JE, Hurlbert RJ, Fehlings MG, Yong VW. Neuroprotection by minocycline facilitates significant recovery from spinal cord injury in mice. Brain 2003;126(Pt 7):1628-1637.

97. Blight AR. Effects of silica on the outcome from experimental spinal cord injury: implication of macrophages in secondary tissue damage. Neuroscience 1994;60:263-273.

98. Jones TB, McDaniel EE, Popovich PG. Inflammatory-mediated injury and repair in the traumatically injured spinal cord. Curr Pharm Des 2005;11:1223-1236.
99. Giulian D, Robertson C. Inhibition of mononuclear phagocytes reduces ischemic injury in the spinal cord. Ann Neurol 1990;27:33-42.

100. Taoka Y, Okajima K, Uchiba M, et al. Reduction of spinal cord injury by administration of iloprost, a stable prostacyclin analog. J Neurosurg 1997;86:1007-1011.

101. Taoka Y, Okajima K, Uchiba M, et al. Role of neutrophils in spinal cord injury in the rat. Neuroscience 1997;79:1177-1182.

102. Pajoohesh-Ganji A, Byrnes KR. Novel neuroinflammatory targets in the chronically injured spinal cord. Neurotherapeutics. doi:10.1007/s13311-011-0036-2.

103. Fleming JC, Bao F, Chen Y, et al. Timing and duration of antialpha4beta1 integrin treatment after spinal cord injury: effect on therapeutic efficacy. J Neurosurg Spine 2009;11:575-587.

104. Lacroix S, Chang L, Rose-John S, Tuszynski MH. Delivery of hyper-interleukin-6 to the injured spinal cord increases neutrophil and macrophage infiltration and inhibits axonal growth. J Comp Neurol 2002;454:213-228

105. Fleming JC, Bao F, Chen Y, Hamilton EF, Relton JK, Weaver LC. Alpha4beta1 integrin blockade after spinal cord injury decreases damage and improves neurological function. Exp Neurol 2008;214:147-159.

106. Bethea JR, Nagashima H, Acosta MC, et al. Systemically administered interleukin-10 reduces tumor necrosis factor-alpha production and significantly improves functional recovery following traumatic spinal cord injury in rats. J Neurotrauma 1999;16:851-863.

107. Brewer KL, Bethea JR, Yezierski RP. Neuroprotective effects of interleukin-10 following excitotoxic spinal cord injury. Exp Neurol 1999;159:484-493.

108. Zhou Z, Peng X, Insolera R, Fink DJ, Mata M. IL-10 promotes neuronal survival following spinal cord injury. Exp Neurol 2009;220:183-190.

109. Lee SI, Jeong SR, Kang YM, et al. Endogenous expression of interleukin-4 regulates macrophage activation and confines cavity formation after traumatic spinal cord injury. J Neurosci Res 2010;88:2409-2419.

110. Huang X, Choi JK, Park SR, et al. GM-CSF inhibits apoptosis of neural cells via regulating the expression of apoptosis-related proteins. Neurosci Res 2007;58:50-57.

111. Bouhy D, Malgrange B, Multon S, et al. Delayed GM-CSF treatment stimulates axonal regeneration and functional recovery in paraplegic rats via an increased BDNF expression by endogenous macrophages. FASEB J 2006;20:1239-1241. 